OPEN ACCESS

Edited by:

Yongsheng Kevin Li, Harbin Medical University, China

Reviewed by: Shengli $\mathrm{Li}$, University of Texas Health Science Center at Houston, United States Zhe Su, The University of Texas at Austin, United States

*Correspondence: Xianzeng Hou houxianzeng@sina.com

Specialty section: This article was submitted to RNA,

a section of the journal Frontiers in Genetics

Received: 29 October 2019 Accepted: 19 December 2019 Published: 28 January 2020

Citation: Wang J, Qi J and Hou X (2020) Systematically Dissecting the Function of RNA-Binding Proteins During Glioma Progression.

Front. Genet. 10:1394. doi: 10.3389/fgene.2019.01394

\section{Systematically Dissecting the Function of RNA-Binding Proteins During Glioma Progression}

\author{
Jianjun Wang ${ }^{1}$, Jianfeng $\mathrm{Qi}^{1,2}$ and Xianzeng Hou ${ }^{1 *}$ \\ ${ }^{1}$ Department of Neurosurgery, The First Hospital Affiliated with Shandong First Medical University, Shandong Provincial \\ Qianfoshan Hospital, Jinan, China, ${ }^{2}$ College of Medicine, Shandong First Medical University, Taian, China
}

RNA-binding proteins (RBPs) play important roles in regulating gene expression and dysregulation of RBPs have been observed in various types of cancer. However, the role of RBPs during glioma progression, and particular in Chinese patients, is only starting to be unveiled. Here, we systematically analyzed the somatic mutation, gene expression patterns of 2949 RBPs during glioma progression. Our comprehensive study reveals several of highly mutated genes (such as ATRX, TTN and SETD2) and differentially expressed genes (such as KIF4A, TTK and CEP55). Integration of the expression of RBPs and genes, we constructed a regulatory network in glioma and revealed the functional links between RBPs and cancer-related genes. Moreover, we identified the prognosis spectrum of RBPs during glioma progression. The expression of a number of RBPs, such as SNRPN and IGF2BP3, are significantly associated with overall survival of patients in all grades. Taken together, our analyses provided a valuable RBP resource during glioma progression, and revealed several candidates that potentially contribute to development of therapeutic targets for glioma.

Keywords: glioma progression, RNA-binding protein, mutations, regulatory network, prognosis

\section{INTRODUCTION}

RNA-binding proteins (RBPs) play crucial roles in post-transcriptional events and perturbations in RBP activity have been associated with various types of cancer (Pereira et al., 2017; Hentze et al., 2018). Understanding the function of RBPs in cancer will help identifying potential prognostic and response biomarkers for design of therapeutic targets (Bonnal et al., 2012; Kudinov et al., 2017). Glioma is a common and aggressive type of brain tumor, which was with poor outcome and no effective treatment by far (Ostrom et al., 2014; Reifenberger et al., 2017). Systematical dissection of RBP functions during glioma progression will provide new insights into the underlying mechanisms of glioma.

Comprehensive identification and annotation of human RBPs are the primary step for investigating their functions. With the development of high throughput sequencing, numbers of RBPs have been identified (Gerstberger et al., 2014). Several databases have curated a number of RBPs. For example, RBPDB is a database for collection of experimentally validated RBPs (Cook et al., 2011). ATtRACT also manually curated approximate 370 RBPs (Giudice et al., 2016). Recently, EuRBPDB has been constructed, which is a widely-used resource for RBPs (Liao et al., 
2019). Moreover, number of studies have found a lot of alterations in RBPs during cancer development and progression. Wang et al., revealed the importance of RBPs in carcinogenesis by large-scale transcriptional profiling studies (Wang et al., 2015). The mutational spectrum of RBPs had been analyzed and identified a number of RBPs exhibited significantly mutation in cancer (Neelamraju et al., 2018). Li et al., have also decoded the genome-wide RBP mutational and transcriptomic landscape and yielded valuable insights into the function of RBPs (Li et al., 2019a). All these results suggest that there are prevalent alterations of RBPs in cancer development and progression.

RBPs have also been demonstrated to play important roles in neurodegeneration and glioma progression (Pereira et al.,, 2017). Numbers of RBPs have also been identified in glioma. Correa et al. revealed the splicing regulator SNRPB as an oncogenic candidate in glioblastoma (GBM) through functional genomics analyses (Correa et al., 2016). RNA-binding protein PCBP2 has been identified to modulate glioma growth by regulating FHL3 (Han et al., 2013). Moreover, Musashil was found to be a central regulator of adhesion pathways in GBM (Uren et al., 2015). The RBP IMP2 can preserve GBM stem cells by preventing let-7 target gene silencing (Pereira et al.,, 2017). Although a number of regulatory networks have been analyzed during glioma progression, such as microRNA-gene regulatory network (Li et al., 2013), transcriptional regulatory network (Li et al., 2015), competitive endogenous RNAs (ceRNAs) network (Xu et al., 2015), we are still lack of knowledge about RBP regulatory network during glioma progression.

To address these questions, we systematically analyzed the genetic and transcriptomic alterations of RBPs during glioma progression. We identified a number of RBPs with somatic mutations, differentially expressed during glioma progression. In addition, several RBPs associated with patient overall survival were also identified. These RBPs regulated a number of cancerrelated genes and played important roles during glioma progression in Chinese patients. All these results provide novel insights into the function of RBPs in glioma.

\section{MATERIALS AND METHODS}

\section{Collection of Human RNA-Binding Proteins}

All the human RBPs were downloaded from the EuRBPDB database, which is a comprehensive resource for annotation of eukaryotic RBPs. In total, there were 2,949 RBPs and these RBPs were further classified into canonical and non-canonical RBPs.

\section{Genetic Alteration Profiles of Glioma}

The genetic alterations for Chinese glioma patients were downloaded from Chinese Glioma Genome Atlas (CGGA). There were 286 patients sequenced by whole-exome sequencing (Hu et al., 2018). We directly downloaded the gene-level mutation datasets. In this table, each row represents one gene and the columns represent the glioma patient. The one values indicated that this gene was mutated in corresponding patient while zeros indicated not mutated. In addition, we also downloaded the clinical information of these patients from CGGA (Table S1). The grade, gender, age, overall survival time, censor status and isocitrate dehydrogenase (IDH) mutation status were included. In addition, we downloaded the somatic mutations of low-grade glioma (LGG) and GBM patients from The Cancer Genome Atlas (TCGA) project. The overall survival and disease-free survival time of these patients were also downloaded.

\section{Genome-Wide Gene Expression Profile of Glioma}

Genome-wide gene expression of glioma patients were also downloaded from CGGA (September 9, 2019). mRNA-Seq data were used in our analyses, which included 693 patients in total. The reads were aligned by STAR (Dobin et al., 2013) and the expression were evaluated as RSEM (Li and Dewey, 2011). There were 185 patients with both somatic mutations and RNASeq data (Figure S1). We also downloaded the clinical information for these 693 patients (Table S2).

\section{Identification of Top Mutated Genes in Glioma}

To identify the genes with high mutation frequency, we separately ranked each gene in grade II, III, and IV glioma. The gene mutation frequency was defined as:

$$
F(g)=\frac{n_{g}}{N}
$$

where $n_{g}$ was the number of patients with gene $g$ mutated and $N$ was the total number of patients in specific grade.

\section{Identified the Genes With Perturbed Expression in Glioma}

We used Wilcoxon's rank sum test to evaluate the difference of gene expression between two adjacent grades. For example, the fold changes for comparison between grade II and III were defined as the (average expression of genes in grade III)/ (average expression of genes in grade II). The $p$-values of Wilcoxon's rank sum test were adjusted by BenjaminiHochberg $(\mathrm{BH})$ method. Genes with fold changes $>2$ and adjusted $p$-values $<0.05$ were defined as up-regulated genes and those with fold changes $<0.5$ and adjusted $p$-values $<0.05$ were defined as down-regulated genes. The comparisons were performed between grade II vs. III, and III vs. IV.

\section{Construction of RNA-Binding Protein- Gene Regulatory Network in Glioma}

RNA-binding proteins are key regulators of gene expression, yet only a small fraction have been functionally characterized (Pereira et al., 2017). It is still difficult to identify the target genes for the majority of RBPs. Increasing studies have demonstrated that the regulators are likely to co-express with their target genes. Thus, we identified the co-expressed genes of $\mathrm{RBPs}$ and constructed the $\mathrm{RBP}$-gene regulatory network in glioma. Here, only the cancer genes were considered and the 
cancer-related genes were downloaded from COSMIC Cancer Gene Census (Oct 25, 2019) (Sondka et al., 2018). There are 723 genes in total. For each RBP-gene pair, we calculated the Pearson correlation coefficient (PCC) as follows:

$$
R_{i j}=\frac{1}{n-1} \sum_{i=1}^{n}\left(\frac{X_{i}-\bar{X}}{\sigma_{X}}\right)\left(\frac{Y_{i}-\bar{Y}}{\sigma_{Y}}\right)
$$

where $\bar{X}$ nd $\bar{Y}$ where the average expression of RBP $X$ and gene $Y$, $\sigma_{X}$ and $\sigma_{Y}$ where the standard error of expression of RBP and gene. There were $n$ patients in the analysis. All the RBP-gene pairs with PCCs $>0.70$ and $p$-values $<0.05$ were identified to construct the regulatory network. The network was visualized by Cytoscape (version 3.7.1) (Shannon et al., 2003).

\section{Identifying Clinical-Associated RNA- Binding Proteins in Glioma}

To identify the RBPs whose expression was potentially correlated with glioma patient survival, we first divided patients in each grade into two groups based on the median expression of each RBP. The survival difference between two groups were evaluated by log-rank test. The hazard ratio (HR) was also calculated. This procedure was performed by the $\mathrm{R}$ package (version 3.6.1) (https://cran.r-project.org/web/packages/survival/index.html). RBPs with $\mathrm{HR}>1$ and $p<0.05$ were defined as risky factors and those with $\mathrm{HR}<1$ and $p<0.05$ were defined as protective factors.

\section{RESULTS}

\section{High-Grade Glioma Patients Exhibit Poor Prognosis and Less Isocitrate Dehydrogenase Mutation}

High-grade glioma remains incurable despite number of genetic alterations have been revealed (Chen et al., 2016). Here, we analyzed the 286 glioma patients sequenced by whole-exome sequencing. We found that glioma patients in high-grade exhibited poor prognosis (Figure 1A, log-rank $p<0.001$ ). Moreover, we explored the 693 patients with mRNA-Seq data. We also found that the patients in high grade were with significantly poorer survival (Figure 1B, log-rank $p<0.001$ ). Particularly, the patients in grade IV (glioblastoma, also known GBM) were with the poorest survival (Figures 1A, B). These results were consistent with the current knowledge, that GBM is the most aggressive cancer.
A

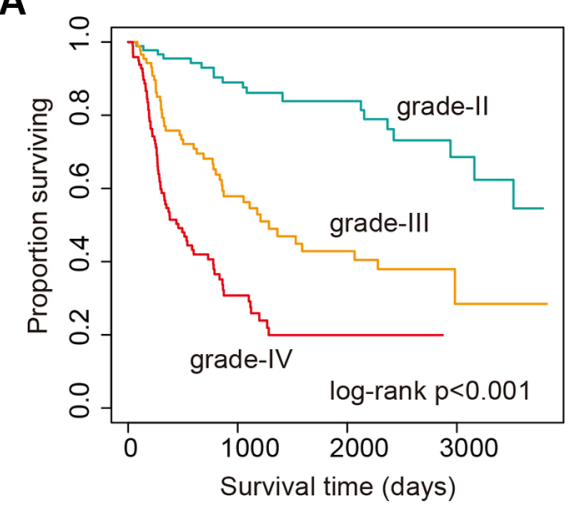

C

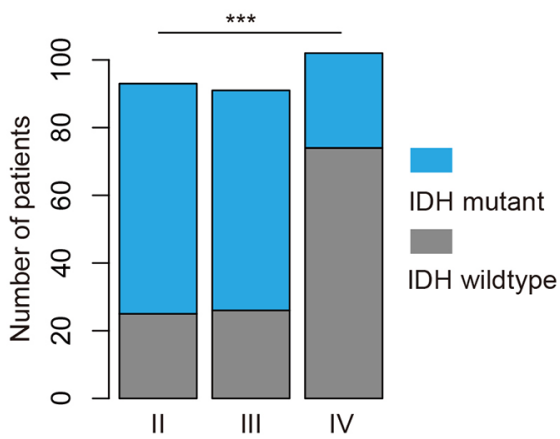

B

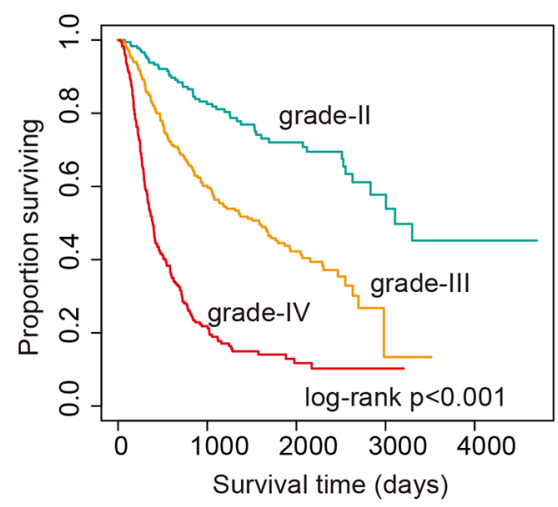

D

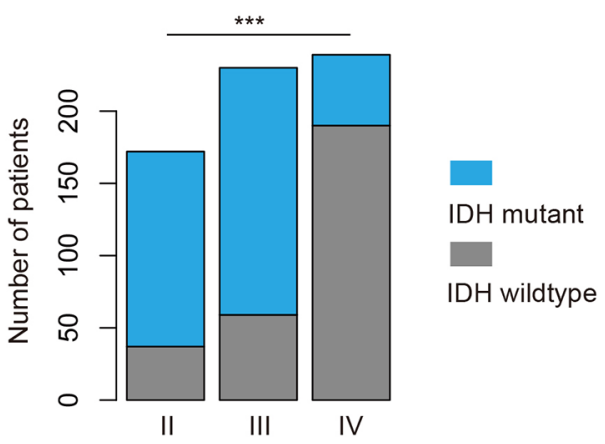

FIGURE 1 | High grade glioma patients with poor survival and less isocitrate dehydrogenase (IDH) mutation. (A) Kaplan-Meier plot indicating survival of glioma patients with mutation data in different grades. (B) Kaplan-Meier plot indicating survival of glioma patients with expression data in different grades. (C) The proportion of patients with IDH mutation or wild type in different grades. Those patients were with mutation data. (D) The proportion of patients with IDH mutation or wild type in different grades. Those patients were with expression data. ${ }^{\star \star} \mathrm{P}<0.001$. 
Next, we investigated whether there are somehow difference in the clinical information for patients in different grades. We first compared the ages of patients with mutation data. The average ages for patients in grade II and III were 38.21 and 39.53 years. The average ages for patients in grade IV were 47.56 , which were significantly older than II and III ( $p$-values $<0.01$, Wilcoxon's rank sum tests). Moreover, we got the similar results in the patients with mRNA data. However, there were no significant difference between grade II and III. IDH1 is the most commonly mutated gene in glioma (Philip et al., 2018). We thus investigated the mutation frequency of IDH1 in glioma patients. We found that GBM patients were with less IDH mutation, either in the exome sequencing cohort or the mRNA-Seq cohort (Figures 1C, D, $p$ values $<0.001$, Fisher's exact test). Moreover, we analyzed the data from TCGA project and found that patients with IDH1 mutation exhibit better overall survival than the wide type ones in LGG and GBM (Figures 2A, C). When considering the disease-free survival time, we found that patients with IDH1 mutation also show better survival in LGG and GBM (Figures 2B, D). All these results suggest that high-grade glioma patients were older, were not likely with IDH1 mutation and exhibited poor survival.

\section{Prevalent Somatic Mutations of RNA- Binding Protein During Glioma Progression}

RBPs have been found to play critical roles in glioma. We thus next investigated the genetic alterations of 2,949 RBPs in glioma (Figure 3A). There were 1,826 (61.92\%) canonical RBPs with specific RNA binding domains, and 1,123 (38.08\%) noncanonical RBPs. Next, we calculated the number of RBPs with different binding domains. We found that there were more than 150 RBPs with RRM_1 domains (Figure 3B). We explored whether each patient was with RBP mutation and found that approximate $87.10 \%$ patients in grade II, $94.50 \%$ patients in grade III and $84.31 \%$ patients in grade IV were with RBP mutations (Figure 3C). These results suggest that there were prevalent somatic mutations in RBPs during glioma progression.

We next explored which RBPs were with higher mutation frequency in different grades glioma patients. Top ranked 10 mutated genes in each grade were shown in Figure 3D. We found that ATRX was ranked top 1 in all grades. The ATRX status has been found to be one of the critical markers that define the molecular classification of gliomas (Nandakumar et al., 2017).
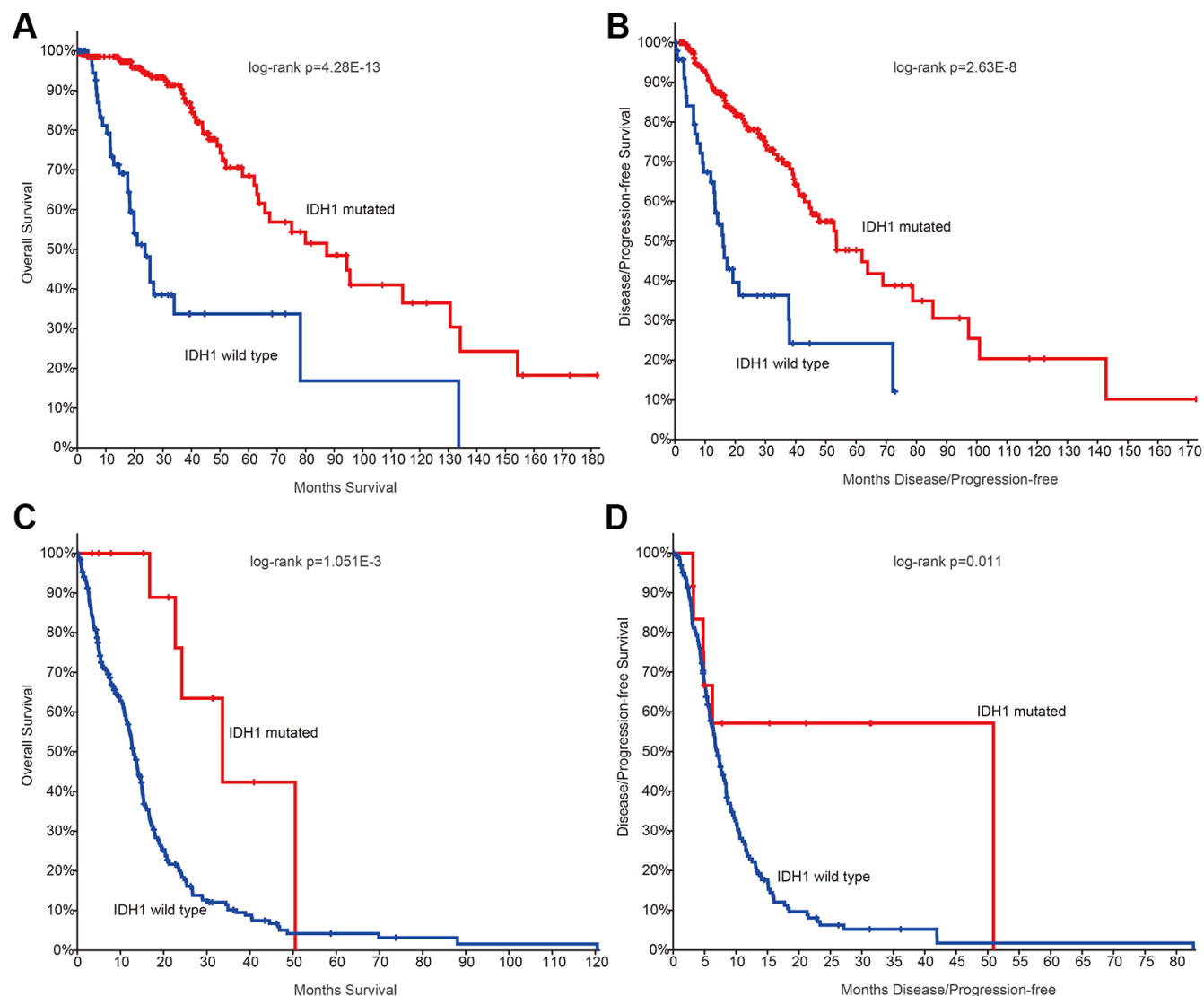

FIGURE 2 | The survival plots for glioma patients in The Cancer Genome Atlas (TCGA) project. (A) Kaplan-Meier plot indicating overall survival of low-grade glioma patients with isocitrate dehydrogenase 1 (IDH1) mutation or not. (B) Kaplan-Meier plot indicating disease-free survival of low-grade glioma patients with IDH1 mutation or not. (C) Kaplan-Meier plot indicating overall survival of glioblastoma (GBM) patients with IDH1 mutation or not. (D) Kaplan-Meier plot indicating diseasefree survival of GBM patients with IDH1 mutation or not. 
A

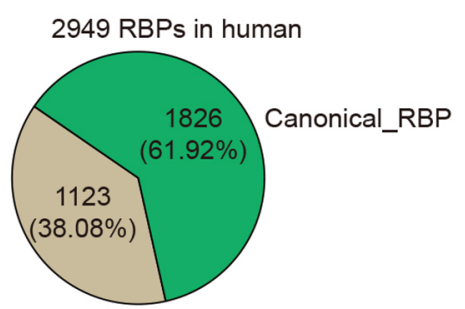

Non-canonical_RBP

C

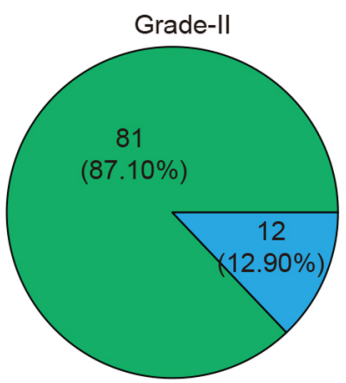

B
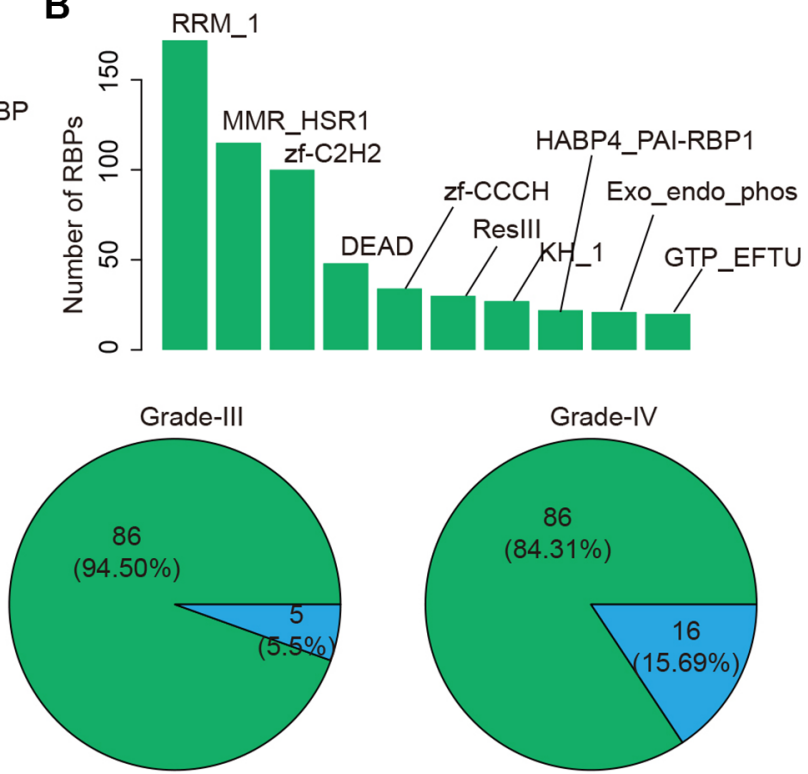

Patients with RBP mutations

Patients without RBP mutations
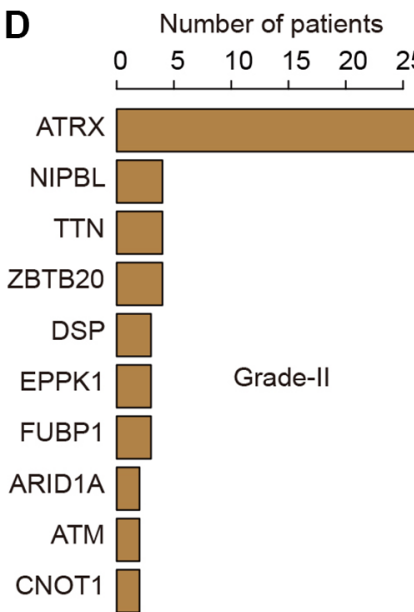

Number of patients

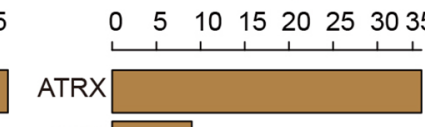

TTN ARID1A

KMT2D

NIPBL

FUBP1

PLEC

AHNAK

HUWE1

SETD1B

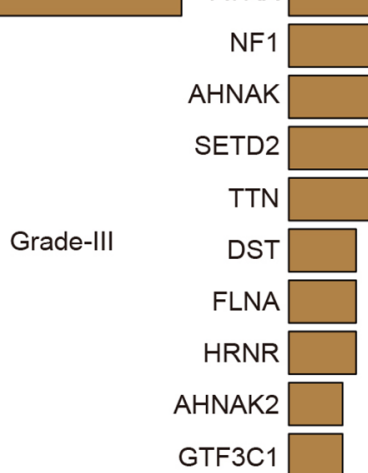

FIGURE 3 | The mutation spectrum of RNA-binding protein (RBP) mutations in glioma. (A) The pie chart shows the proportion of canonical and non-canonical RBPs. (B) The bar charts shows the number of RBPs in top ranked RBP families. (C) The pie charts show the proportion of patients in different grade with RBP mutations. Left for grade II, middle for grade III and right for grade IV. (D) Top ranked 10 genes by mutation frequency in different grades. Left for grade II, middle for grade III and right for grade IV.

ATRX loss can promote tumor growth and impair DNA repair in glioma (Koschmann et al., 2016). In total, we found that ATRX1 was mutated in approximate $30 \%$ of all glioma patients (Figure 4). Another frequently mutated gene was TTN in all grades (Figure 3D), which was also identified previously in glioma (Panossian et al., 2018). TTN was mutated in $6 \%$ of all glioma patients (Figure 4). Moreover, we found that NF1 were with higher mutation frequency in GBM, which has been used to define the mesenchymal subtype of GBM (Verhaak et al., 2010). We also identified several candidate genes, such as ARID1A, SETD2, FLNA and KMT2D. In addition, we queried the PubMed and found that numbers of these genes were co-occurred with "glioma" or "glioblastoma" in literature (Figure S2 and S3). These results provided candidate RBPs for further functional investigation in glioma.

\section{Expression Perturbations of RNA-Binding Proteins During Glioma Progression}

Besides genetic alterations, evidence have suggested that the expression of RBPs were also perturbed in cancer (Sebestyen et al., 2016; Li et al., 2017). We next systematically analyzed the RBP transcriptome in different grades of glioma. We identified 


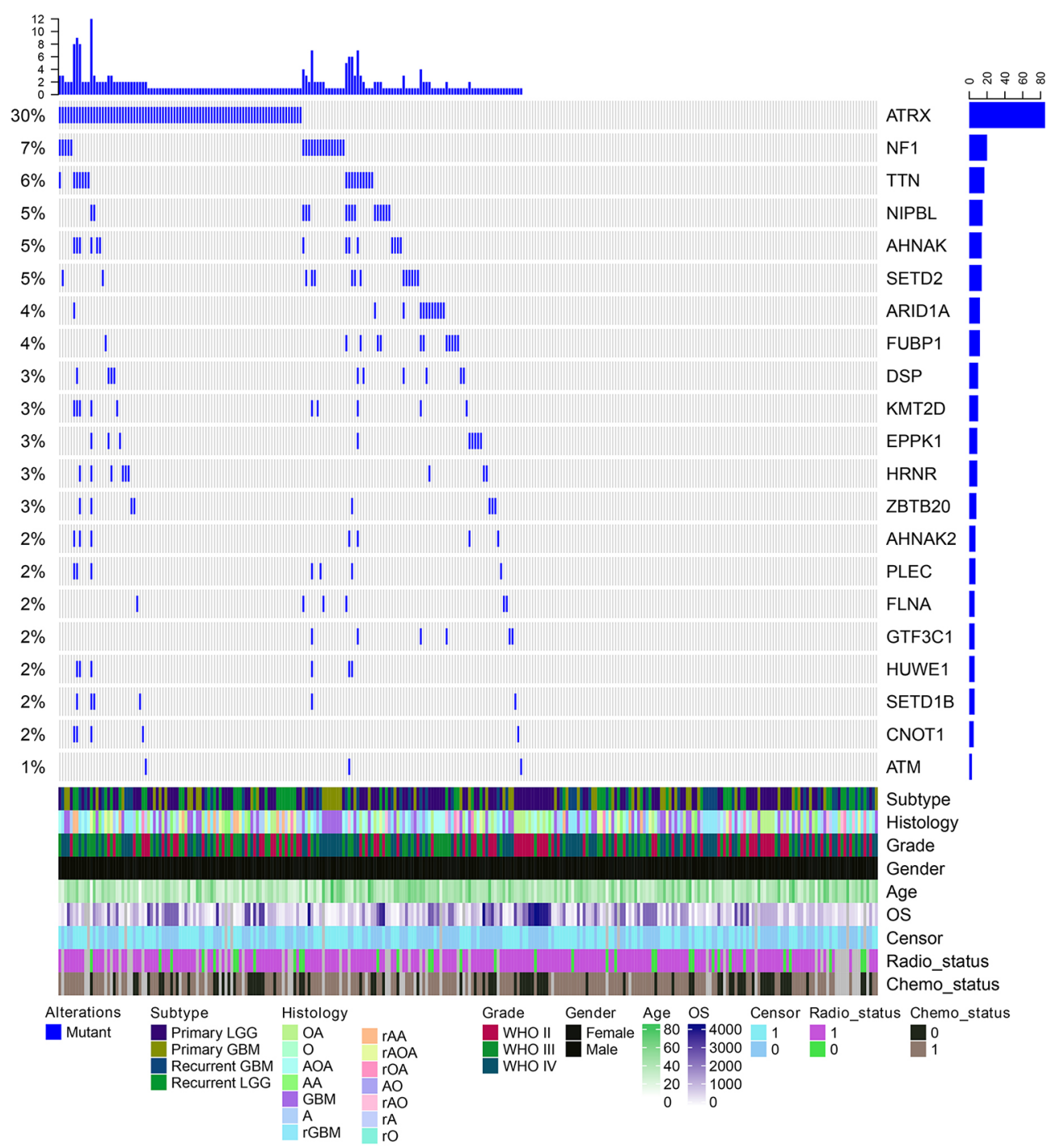

FIGURE 4 | The mutation spectrum of top ranked RNA-binding proteins (RBPs) in glioma. Each column is one glioma patient. The blue lines indicate whether the gene is mutated or not. The clinical information of these patients are shown on the bottom panels.

32 up-regulated RBPs in comparison between grade II vs. III (Figure 5A). There are more RBPs exhibited expression perturbations when comparison between grade III and IV (Figure 5B and Table S3). These results suggested that the transcriptome were likely to be perturbed during the progression from low grade to high grade. Among the top up-regulated genes in comparison between grade II and III, we identified four important genes, such as IGF2BP2, TTK, KIF4A and CEP55 (Figure 5C). It has been shown that IGF2BP2 was a direct target of miR-188 in glioma, and IGF2BP2 under-expression served tumor-suppressive roles in glioma growth and metastasis (Ding et al., 2017). CEP55 has been found to promote cell proliferation and inhibits cell apoptosis in glioma (Li et al., 2018b).

When we compared the transcriptome of patients in grade III vs. grade IV, we identified 56 up-regulated genes and
12 down-regulated genes (Figure 5B). Among the up-regulated genes, we identified NNMT, LGALS3, PDLIM4, TUBA1C and ANXA2 as top five (Figure 5D). NNMT silencing had been shown to activate tumor suppressor PP2A and inhibits tumor forming (Palanichamy et al., 2017). This was consistent with our result that it was up-regulated in GBM. LGALS3 was also found to promote GBM and was associated with tumor risk and prognosis (Wang et al., 2019). PDLIM4 had been identified as a gene signature associated with the clinical outcome in high-grade gliomas (de Tayrac et al., 2011). For the downregulated genes, we have identified CSDC2, ZNF804B, LIN28A and SRRM3 as candidates. However, few of these were investigated in current studies. These results suggested that the tumor suppressors need to be paid attention in future glioma studies. Taken together, function analysis of these 


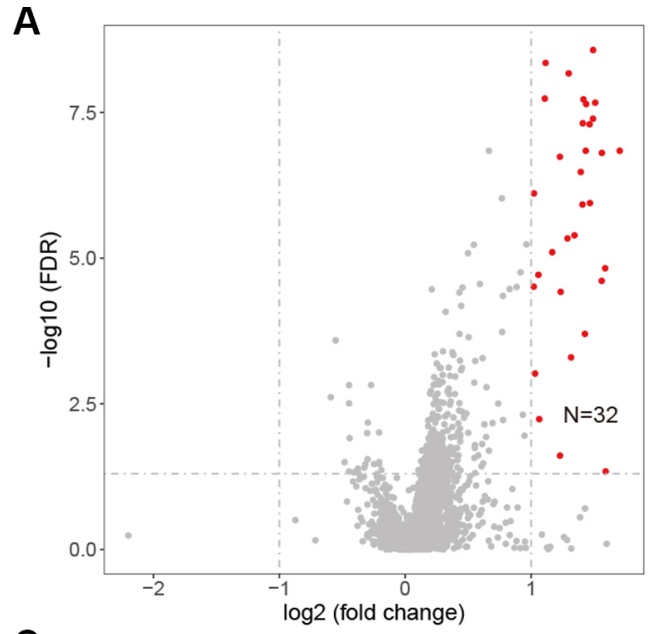

C

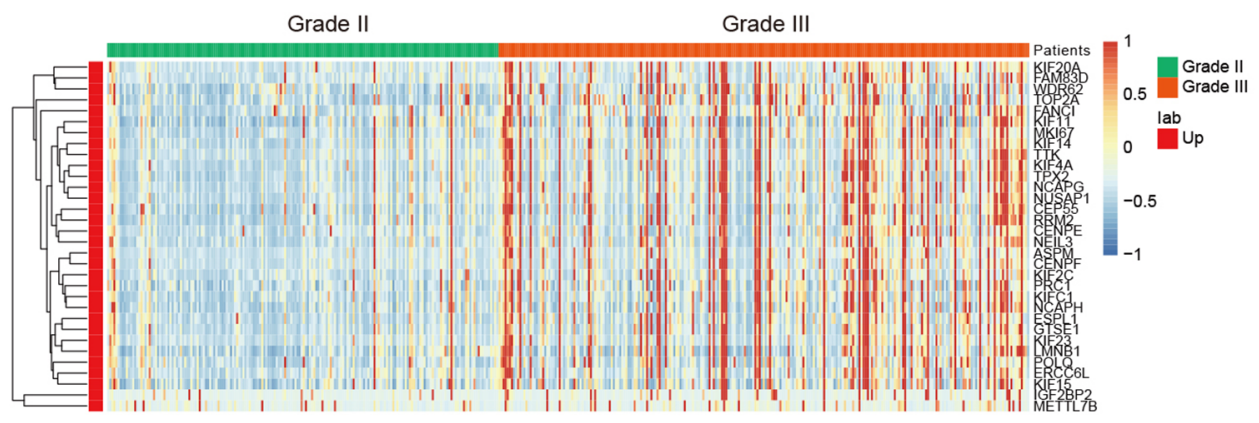

D

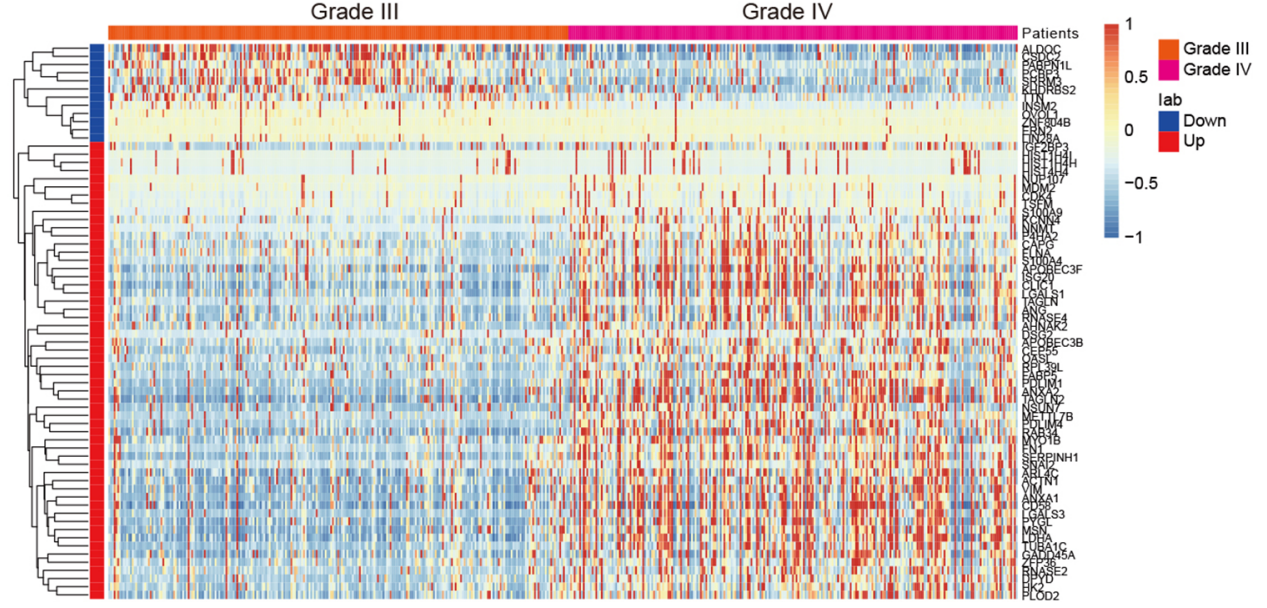

FIGURE 5 | Differentially expressed genes in glioma. (A) Volcano plot shows the differentially expressed genes between grade II and III. Red for up-regulated genes. (B) Volcano plot shows the differentially expressed genes between grade III and IV. Red for up-regulated genes and blue for down-regulated genes. (C) Heat map for genes that perturbed between grade II and III. (D) Heat map for genes that perturbed between grade III and IV.

RBPs provide insight into the transcriptome perturbations of glioma progression.

\section{RNA-Binding Protein Regulatory Network During Glioma Progression}

Proteins do not function isolatedly but interact with other molecules in complex cellular networks for signal transduction (Xu et al., 2017; Yi et al., 2017). Understanding the RBP

B

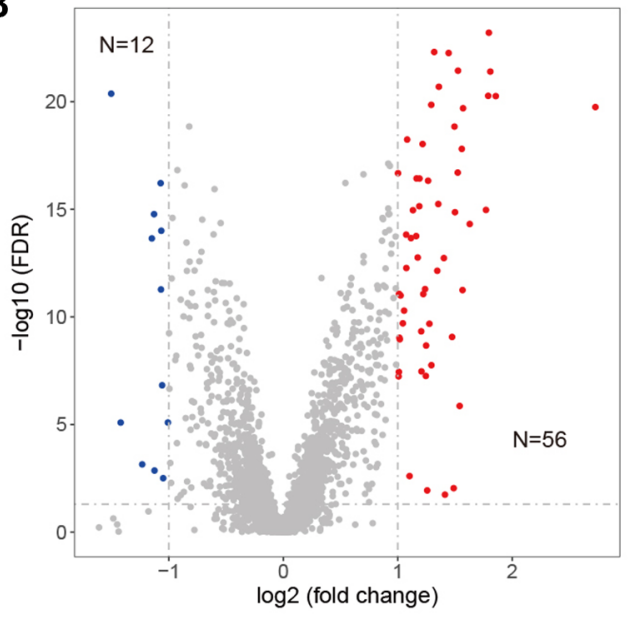

Grade III 
A

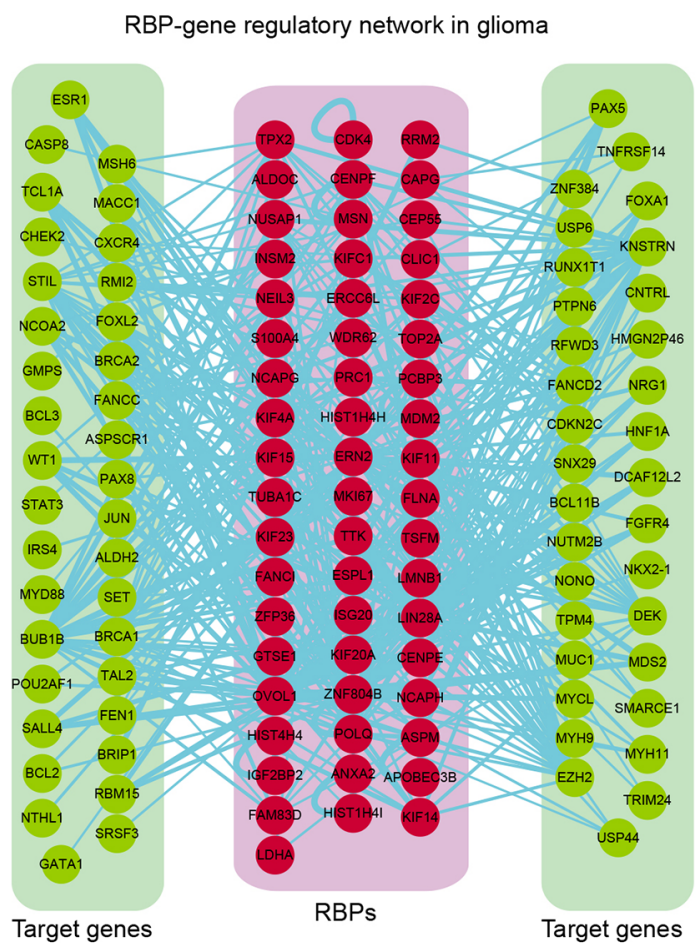

B

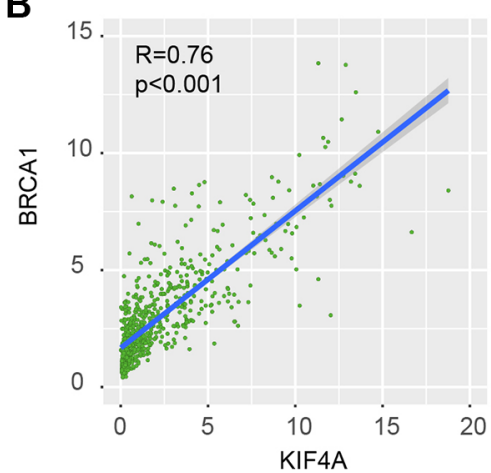

C

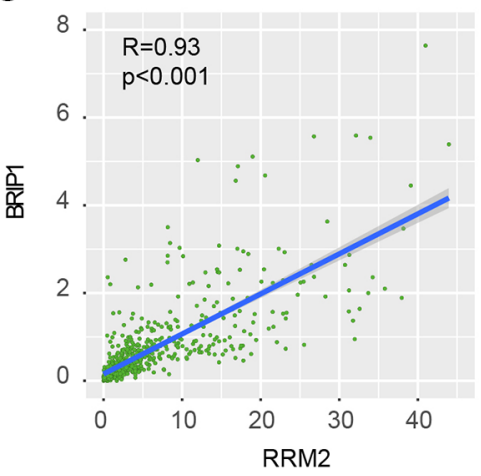

FIGURE 6 | RNA-binding protein (RBP)-gene regulatory network in glioma. (A) The RBP-gene regulatory network in glioma. Red for RBPs and green for target genes that were related to cancer. (B) Scatter plot shows the correlation between KIF4A and BRCA1 in glioma. (C) Scatter plot shows the correlation between RRM2 and BRIP1 in glioma.

was correlated with cell cycle, G2M checkpoint (Cho et al., 2019). Abrogation of BRCA1 had also been found to play roles in tumor growth in glioma (Rasmussen et al., 2016). We found that the expression of KIF4A and BRCA1 was significantly correlated with each other in glioma (Figure 6B, $R=0.76$ and $p$-value < 0.001 ), providing a functional link between RBP and gene.

Another example was RRM2 and BRIP1, which was significantly correlated with each other in expression (Figure 6C, $R=0.93$ and $p$-value $<0.001)$. RRM2 had been found to promote the progression of human GBM and was a potential prognostic biomarker in glioma (Li et al., 2018a; Sun et al., 2019). BRIP1 was found to be an independent signature, which was correlated with worse prognosis in glioma (de Sousa et al., 2017). Our results provided a way to functionally explain the signaling of RRM2 during glioma progression. Moreover, we also identified the functional association between TTK and BUB1B, KIF23 and POLQ. All these RBP-gene correlations provide suitable ways for functional characterization of RBPs in glioma.

\section{Prognostic Potential of RNA-Binding Protein Regulators}

RBPs are essential modulators of transcription and numbers of RBPs have being found to be associated with the survival of patients (Frau et al., 2013). We next identified the RBPs that were associated with the survival of patients in different grades. We found that there were more RBPs were associated with survival in grade III, compared with other two grades (Figure 7 and Table S6). For the protective RBPs in glioma, 25, 425 and 28 RBPs were only associated survival in grade II, III and IV. Three RBPs (ARPP21, SNRPN and GLRX3) were associated with patient overall survival in all grades (Figure 7A). SNRPN had been found as a autism-related gene by regulating cortical and spine development via nuclear receptor (Li et al., 2016). Hypermethylation of SNRPN increased as the cellular origin of the tumors advanced in oogenesis and was closely correlated in individual teratomas (Miura et al., 1999). We found that the high expression of SNRPN was correlated with better overall survival in all grade glioma patients (Figures 7B-D, log-rank $p$-values $<0.05$ ).

In addition, we also identified numbers of risky RBPs in glioma (Figure 7E). There were 8, 1,220, 12 RBPs were associated with survival in specific grade. In total, 27 RBPs were identified as risky factors in all grades, including BRCA1, MCM2, IGF2BP3, KIF2C, VIM and PLOD3. High MCM2 expression was found to be strongly associated with poor overall survival in patients with high-grade glioma in our current study, as well as previous studies (Hua et al., 2014). Recently, IGF2BP3 has been identified as a potential oncogene across multiple cancer types ( $\mathrm{Li}$ et al., 2019b). We found that the high expression of IGF2BP3 was significantly associated with poor prognosis in all three grades (Figure $7 \mathbf{F}-\mathbf{H}, \log$-rank $p$-values $<0.05$ ). These results provided 


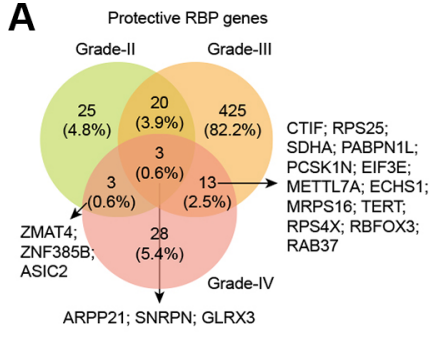

E

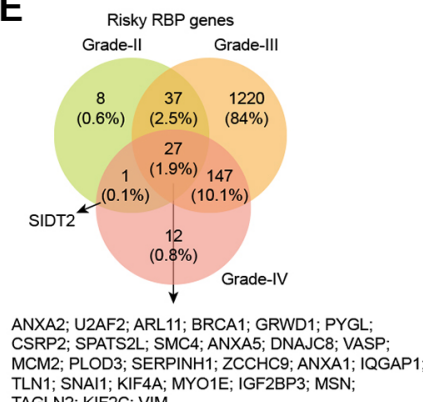

B

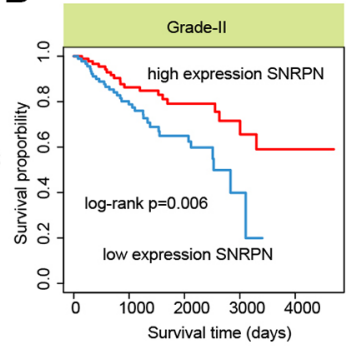

$\mathbf{F}$

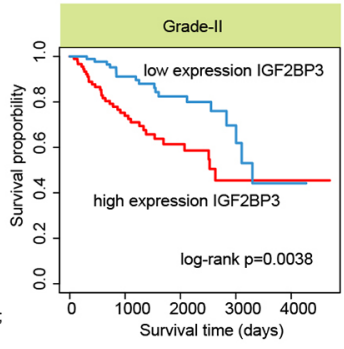

C

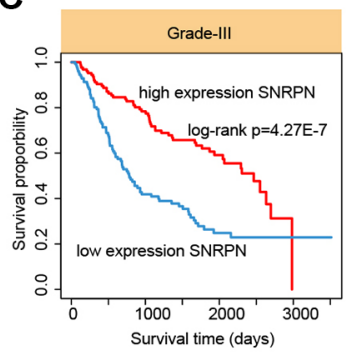

G

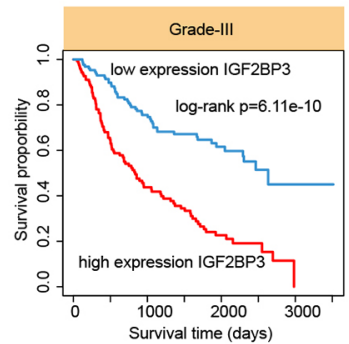

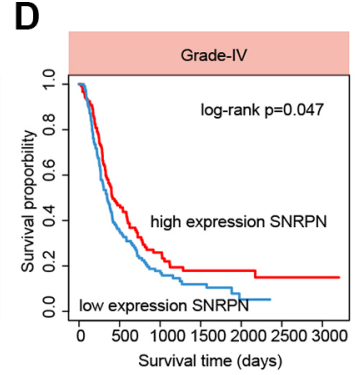

H

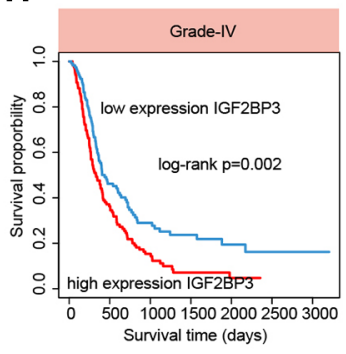

FIGURE 7 | The survival landscape of RNA-binding proteins (RBPs) in glioma. (A) Venny plots show the protective RBPs in different grade glioma patients. (B-D) Kaplan-Meier plots indicating overall survival of glioma patients with low and high expression of SNRPN. (E) Venny plots show the risky RBPs in different grade glioma patients. (F-H) Kaplan-Meier plots indicating overall survival of glioma patients with low and high expression of IGF2BP3.

more evidence for the oncogene roles of IGF2BP3 in cancer. Taken together, our analyses provided a prognostic spectrum for RBPs during glioma progression.

\section{DISCUSSION}

In this study, we systematically analyzed the genetic and transcriptome alterations of RBPs during glioma progression. The top mutated RBPs in different grades of glioma patients were identified and several of them had been found to play important roles in glioma or other cancers. Moreover, we compared the transcriptome and identified the differentially expressed RBPs. We found that there were more RBPs exhibited expression perturbations during the transition from grade III to IV. These results suggested that the transcriptome was greatly perturbed in the progression of high-grade glioma. Our regulatory network and prognosis analysis also revealed several important candidates for functional characterization in future experiments.

Although several candidate RBPs were identified in our current study, there are a lot of work need to do for investigating the detail functional ways of these RBPs. RBPs have been found to regulate alterative splicing (AS) and influences the expression of genes (Fei et al., 2017). Alterations of AS are emerging as important signatures in cancer (Liu et al., 2017). The mutation of RBPs generally impair the recognition of regulatory sites, and affecting the splicing of multiple genes. However, it is still difficult to determine the targets for the majority of RBPs. The best method for identifying the targets of RBPs is CLIP-Seq, but there are limited number of data currently. With the development of sequencing technology, such as CLIP-Seq, we will get more details about the function of RBPs. Moreover, RBPs can also interact with noncoding RNAs. Identifying the cell type specific RBP interactome will yield novel insight into the function of RBPs.

Moreover, we identified an important RBP IGF2BP3 during glioma progression. Beside RBP, this genes also an epigenetic regulator, which can affect the fates of mRNA in an m6Adependent manner (Bi et al., 2019). These results suggest that m6A might also play important roles during glioma progression. Several studies have emerged to reveal the function of $\mathrm{m} 6 \mathrm{~A}$ in glioma (Dixit et al., 2017; Zhang et al., 2017). But we are still lack of knowledge about the landscape of m6A alterations in glioma, particular in Chinese cohort. Moreover, we are not sure to what extent RBPs can regulate m6A. Is it just one case or general regulation? With the increasing data of RBP regulation as well as other epigenetic data, we will get deep insight into this regulatory layer in cancer.

In summary, our comprehensive analyses dissect the potential function of RBPs during glioma progression. Understanding the functions of candidate RBPs identified in this study will provide insight into the underlying mechanisms of glioma progression.

\section{DATA AVAILABILITY STATEMENT}

Publicly available datasets were analyzed in this study. This data can be found here: http://www.cgga.org.cn/download.jsp. 


\section{AUTHOR CONTRIBUTIONS}

JW contributed to data analysis and paper writing, JQ contributed to figure construction, $\mathrm{XH}$ contributed to the design, data analysis and paper writing.

\section{FUNDING}

This paper was supported by grant 2015BSB14042 from the Natural Science Foundation of Shandong Province, China.

\section{REFERENCES}

Bi, Z., Liu, Y., Zhao, Y., Yao, Y., Wu, R., Liu, Q., et al. (2019). A dynamic reversible RNA N(6) -methyladenosine modification: current status and perspectives. J. Cell. Physiol. 234, 7948-7956. doi: 10.1002/jcp.28014

Bonnal, S., Vigevani, L., and Valcarcel, J. (2012). The spliceosome as a target of novel antitumour drugs. Nat. Rev. Drug Discovery 11, 847-859. doi: 10.1038/ nrd3823

Chen, R., Cohen, A. L., and Colman, H. (2016). Targeted therapeutics in patients with high-grade gliomas: past, present, and future. Curr. Treat. Options Oncol. 17 (8), 42. doi: 10.1007/s11864-016-0418-0

Cho, S. Y., Kim, S., Kim, G., Singh, P., and Kim, D. W. (2019). Integrative analysis of KIF4A, 9, 18A, and 23 and their clinical significance in low-grade glioma and glioblastoma. Sci. Rep. 9, 4599. doi: 10.1038/s41598-018-37622-3

Cook, K. B., Kazan, H., Zuberi, K., Morris, Q., and Hughes, T. R. (2011). RBPDB: a database of RNA-binding specificities. Nucleic Acids Res. 39, D301-D308. doi: 10.1093/nar/gkq1069

Correa, B. R., de Araujo, P. R., Qiao, M., Burns, S. C., Chen, C., Schlegel, R., et al. (2016). Functional genomics analyses of RNA-Binding Proteins reveal the splicing regulator SNRPB as an oncogenic candidate in glioblastoma. Genome Biol. 17, 125. doi: 10.1186/s13059-016-0990-4

de Sousa, J. F., Torrieri, R., Serafim, R. B., Di Cristofaro, L. F., Escanfella, F. D., Ribeiro, R., et al. (2017). Expression signatures of DNA repair genes correlate with survival prognosis of astrocytoma patients. Tumour Biol. J. Int. Soc. Oncodev. Biol. Med. 39 (4), 1010428317694552. doi: 10.1177/ 1010428317694552

de Tayrac, M., Aubry, M., Saikali, S., Etcheverry, A., Surbled, C., Guenot, F., et al. (2011). A 4-gene signature associated with clinical outcome in high-grade gliomas. Clin. cancer Res. an Off. J. Am. Assoc. Cancer Res. 17, 317-327. doi: 10.1158/1078-0432.CCR-10-1126

Ding, L., Wang, L., and Guo, F. (2017). microRNA188 acts as a tumour suppressor in glioma by directly targeting the IGF2BP2 gene. Mol. Med. Rep. 16, 71247130. doi: $10.3892 / \mathrm{mmr} .2017 .7433$

Dixit, D., Xie, Q., Rich, J. N., and Zhao, J. C. (2017). Messenger RNA methylation Regulates glioblastoma tumorigenesis. Cancer Cell. 31, 474-475. doi: 10.1016/ j.ccell.2017.03.010

Dobin, A., Davis, C. A., Schlesinger, F., Drenkow, J., Zaleski, C., Jha, S., et al. (2013). STAR: ultrafast universal RNA-seq aligner. Bioinformatics 29, 15-21. doi: 10.1093/bioinformatics/bts635

Fei, T., Chen, Y., Xiao, T., Li, W., Cato, L., Zhang, P., et al. (2017). Genome-wide CRISPR screen identifies HNRNPL as a prostate cancer dependency regulating RNA splicing. Proc. Nat. Acad. Sci. U. S. A. 114, E5207-E5215. doi: 10.1073/ pnas. 1617467114

Frau, M., Feo, F., and Pascale, R. M. (2013). Pleiotropic effects of methionine adenosyltransferases deregulation as determinants of liver cancer progression and prognosis. J. Hepatol. 59, 830-841. doi: 10.1016/j.jhep. 2013.04 .031

Gerstberger, S., Hafner, M., and Tuschl, T. (2014). A census of human RNABinding Proteins. Nat. Rev. Genet. 15, 829-845. doi: 10.1038/nrg3813

Giudice, G., Sanchez-Cabo, F., Torroja, C., and Lara-Pezzi, E. (2016). ATtRACT-a database of RNA-Binding Proteins and associated motifs. Database. J. Biol. Databases Curation. doi: 10.1093/ database/baw035

\section{ACKNOWLEDGMENTS}

We would like to thank the CGGA project for providing the datasets of glioma.

\section{SUPPLEMENTARY MATERIAL}

The Supplementary Material for this article can be found online at: https://www.frontiersin.org/articles/10.3389/fgene.2019. 01394/full\#supplementary-material

Han, W., Xin, Z., Zhao, Z., Bao, W., Lin, X., Yin, B., et al. (2013). RNA-binding protein PCBP2 modulates glioma growth by regulating FHL3. J. Clin. Invest. 123, 2103-2118. doi: 10.1172/JCI61820

Hentze, M. W., Castello, A., Schwarzl, T., and Preiss, T. (2018). A brave new world of RNA-Binding Proteins. Nat. Rev. Mol. Cell Biol. 19, 327-341. doi: 10.1038/ nrm.2017.130

Hu, H., Mu, Q., Bao, Z., Chen, Y., Liu, Y., Chen, J., et al. (2018). Mutational landscape of secondary glioblastoma guides MET-targeted trial in brain tumor. Cell 175, 1665-1678 e1618. doi: 10.1016/j.cell.2018.09.038

Hua, C., Zhao, G., Li, Y., and Bie, L. (2014). Minichromosome Maintenance (MCM) family as potential diagnostic and prognostic tumor markers for human gliomas. BMC Cancer 14, 526. doi: 10.1186/1471-2407-14-526

Koschmann, C., Calinescu, A. A., Nunez, F. J., Mackay, A., Fazal-Salom, J. Thomas, D., et al. (2016). ATRX loss promotes tumor growth and impairs nonhomologous end joining DNA repair in glioma. Sci. Transl. Med. 8, 328ra328. doi: 10.1126/scitranslmed.aac8228

Kudinov, A. E., Karanicolas, J., Golemis, E. A., and Boumber, Y. (2017). Musashi RNA-Binding Proteins as cancer drivers and novel therapeutic targets. Clin. Cancer Res. an Off. J. Am. Assoc. Cancer Res. 23, 2143-2153. doi: 10.1158/10780432.CCR-16-2728

Li, B., and Dewey, C. N. (2011). RSEM: accurate transcript quantification from RNA-Seq data with or without a reference genome. BMC Bioinform. 12, 323. doi: 10.1186/1471-2105-12-323

Li, Y., Xu, J., Chen, H., Bai, J., Li, S., Zhao, Z., et al. (2013). Comprehensive analysis of the functional microRNA-mRNA regulatory network identifies miRNA signatures associated with glioma malignant progression. Nucleic Acids Res. 41, e203. doi: 10.1093/nar/gkt1054

Li, Y., Shao, T., Jiang, C., Bai, J., Wang, Z., Zhang, J., et al. (2015). Construction and analysis of dynamic transcription factor regulatory networks in the progression of glioma. Sci. Rep. 5, 15953. doi: 10.1038/srep15953

Li, H., Zhao, P., Xu, Q., Shan, S., Hu, C., Qiu, Z., et al. (2016). The autism-related gene SNRPN regulates cortical and spine development via controlling nuclear receptor Nr4a1. Sci. Rep. 6, 29878. doi: 10.1038/srep29878

Li, Y., Sahni, N., Pancsa, R., McGrail, D. J., Xu, J., Hua, X., et al. (2017). Revealing the determinants of widespread alternative splicing perturbation in cancer. Cell Rep. 21, 798-812. doi: 10.1016/j.celrep.2017.09.071

Li, C., Zheng, J., Chen, S., Huang, B., Li, G., Feng, Z., et al. (2018a). RRM2 promotes the progression of human glioblastoma. J. Cell. Physiol. 233, 67596767. doi: 10.1002/jcp.26529

Li, F., Jin, D., Tang, C., and Gao, D. (2018b). CEP55 promotes cell proliferation and inhibits apoptosis via the PI3K/Akt/p21 signaling pathway in human glioma U251 cells. Oncol. Lett. 15, 4789-4796. doi: 10.3892/ol.2018.7934

Li, Y., McGrail, D. J., Xu, J., Li, J., Liu, N. N., Sun, M., et al. (2019a). MERIT: systematic analysis and characterization of mutational effect on RNA interactome topology. Hepatology 70, 532-546. doi: 10.1002/hep.30242

Li, Y., Xiao, J., Bai, J., Tian, Y., Qu, Y., Chen, X., et al. (2019b). Molecular characterization and clinical relevance of $\mathrm{m}(6) \mathrm{A}$ regulators across 33 cancer types. Mol. Cancer 18, 137. doi: 10.1186/s12943-019-1066-3

Liao, J. Y., Yang, B., Zhang, Y. C., Wang, X. J., Ye, Y., Peng, J. W., et al. (2019). EuRBPDB: a comprehensive resource for annotation, functional and oncological investigation of eukaryotic RNA Binding Proteins (RBPs). Nucleic Acids Res. doi: 10.1093/nar/gkz823 
Liu, J., Liu, T., Wang, X., and He, A. (2017). Circles reshaping the RNA world: from waste to treasure. Mol. Cancer 16, 58. doi: 10.1186/s12943-017-0630-y

Miura, K., Obama, M., Yun, K., Masuzaki, H., Ikeda, Y., Yoshimura, S., et al. (1999). Methylation imprinting of H19 and SNRPN genes in human benign ovarian teratomas. Am. J. Hum. Genet. 65, 1359-1367. doi: 10.1086/302615

Nandakumar, P., Mansouri, A., and Das, S. (2017). The role of ATRX in glioma biology. Front. Oncol. 7, 236. doi: 10.3389/fonc.2017.00236

Neelamraju, Y., Gonzalez-Perez, A., Bhat-Nakshatri, P., Nakshatri, H., and Janga, S. C. (2018). Mutational landscape of RNA-Binding Proteins in human cancers. RNA Biol. 15, 115-129. doi: 10.1080/15476286.2017.1391436

Ostrom, Q. T., Bauchet, L., Davis, F. G., Deltour, I., Fisher, J. L., Langer, C. E., et al. (2014). The epidemiology of glioma in adults: a "state of the science" review. Neuro-oncology 16, 896-913. doi: 10.1093/neuonc/nou087

Palanichamy, K., Kanji, S., Gordon, N., Thirumoorthy, K., Jacob, J. R., Litzenberg, K. T., et al. (2017). NNMT silencing activates tumor suppressor PP2A, inactivates oncogenic STKs, and inhibits tumor forming ability. Clin. Cancer Res. an Off. J. Am. Assoc. Cancer Res. 23, 2325-2334. doi: 10.1158/1078-0432.CCR-16-1323

Panossian, A., Seo, E. J., and Efferth, T. (2018). Novel molecular mechanisms for the adaptogenic effects of herbal extracts on isolated brain cells using systems biology. Phytomed. Int. J. Phytother. Phytopharmacol. 50, 257-284. doi: 10.1016/j.phymed.2018.09.204

Pereira, B., Billaud, M., and Almeida, R. (2017). RNA-Binding Proteins in cancer: old players and new actors. Trends Cancer 3, 506-528. doi: 10.1016/ j.trecan.2017.05.003

Philip, B., Yu, D. X., Silvis, M. R., Shin, C. H., Robinson, J. P., Robinson, G. L., et al. (2018). Mutant IDH1 promotes glioma formation in vivo. Cell Rep. 23, 15531564. doi: 10.1016/j.celrep.2018.03.133

Rasmussen, R. D., Gajjar, M. K., Tuckova, L., Jensen, K. E., Maya-Mendoza, A., Holst, C. B., et al. (2016). BRCA1-regulated RRM2 expression protects glioblastoma cells from endogenous replication stress and promotes tumorigenicity. Nat. Commun. 7, 13398. doi: 10.1038/ncomms 13398

Reifenberger, G., Wirsching, H. G., Knobbe-Thomsen, C. B., and Weller, M. (2017). Advances in the molecular genetics of gliomas - implications for classification and therapy. Nat. Rev. Clin. Oncol. 14, 434-452. doi: 10.1038/ nrclinonc.2016.204

Sebestyen, E., Singh, B., Minana, B., Pages, A., Mateo, F., Pujana, M. A., et al. (2016). Large-scale analysis of genome and transcriptome alterations in multiple tumors unveils novel cancer-relevant splicing networks. Genome Res. 26, 732-744. doi: 10.1101/gr.199935.115

Shannon, P., Markiel, A., Ozier, O., Baliga, N. S., Wang, J. T., Ramage, D., et al. (2003). Cytoscape: a software environment for integrated models of biomolecular interaction networks. Genome Res. 13, 2498-2504. doi: $10.1101 /$ gr. 1239303

Sondka, Z., Bamford, S., Cole, C. G., Ward, S. A., Dunham, I., and Forbes, S. A. (2018). The COSMIC cancer gene census: describing genetic dysfunction across all human cancers. Nat. Rev. Cancer 18, 696-705. doi: 10.1038/ s41568-018-0060-1

Sun, H., Yang, B., Zhang, H., Song, J., Zhang, Y., Xing, J., et al. (2019). RRM2 is a potential prognostic biomarker with functional significance in glioma. Int. J. Biol. Sci. 15, 533-543. doi: 10.7150/ijbs.30114

Uren, P. J., Vo, D. T., de Araujo, P. R., Potschke, R., Burns, S. C., Bahrami-Samani, E., et al. (2015). RNA-Binding Protein musashil is a central regulator of adhesion pathways in glioblastoma. Mol. Cell. Biol. 35, 2965-2978. doi: 10.1128/ MCB.00410-15

Verhaak, R. G., Hoadley, K. A., Purdom, E., Wang, V., Qi, Y., Wilkerson, M. D., et al. (2010). Integrated genomic analysis identifies clinically relevant subtypes of glioblastoma characterized by abnormalities in PDGFRA, IDH1, EGFR, and NF1. Cancer Cell. 17, 98-110. doi: 10.1016/j.ccr.2009.12.020

Wang, J., Liu, Q., and Shyr, Y. (2015). Dysregulated transcription across diverse cancer types reveals the importance of RNA-binding protein in carcinogenesis. BMC Genomics 16 Suppl 7, S5. doi: 10.1186/1471-2164-16-S7-S5

Wang, H., Song, X., Huang, Q., Xu, T., Yun, D., Wang, Y., et al. (2019). LGALS3 promotes treatment resistance in glioblastoma and is associated with tumor risk and prognosis. Cancer Epidemiol. Biomarkers Prev. Publ. Am. Assoc. Cancer Res. cosponsored by Am. Soc. Prev. Oncol. 28, 760-769. doi: 10.1158/ 1055-9965.EPI-18-0638

Xu, J., Li, Y., Lu, J., Pan, T., Ding, N., Wang, Z., et al. (2015). The mRNA related ceRNA-ceRNA landscape and significance across 20 major cancer types. Nucleic Acids Res. 43, 8169-8182. doi: 10.1093/nar/gkv853

Xu, J., Shao, T., Ding, N., Li, Y., and Li, X. (2017). miRNA-miRNA crosstalk: from genomics to phenomics. Brief. Bioinform. 18, 1002-1011. doi: 10.1093/bib/ bbw073

Yi, S., Lin, S., Li, Y., Zhao, W., Mills, G. B., and Sahni, N. (2017). Functional variomics and network perturbation: connecting genotype to phenotype in cancer. Nat. Rev. Genet. 18, 395-410. doi: 10.1038/nrg.2017.8

Zhang, S., Zhao, B. S., Zhou, A., Lin, K., Zheng, S., Lu, Z., et al. (2017). m(6)A Demethylase ALKBH5 maintains tumorigenicity of glioblastoma stem-like cells by sustaining FOXM1 expression and cell proliferation program. Cancer Cell. 31, 591-606 e596. doi: 10.1016/j.ccell.2017.02.013

Conflict of Interest: The authors declare that the research was conducted in the absence of any commercial or financial relationships that could be construed as a potential conflict of interest.

Copyright $\odot 2020$ Wang, Qi and Hou. This is an open-access article distributed under the terms of the Creative Commons Attribution License (CC BY). The use, distribution or reproduction in other forums is permitted, provided the original author(s) and the copyright owner(s) are credited and that the original publication in this journal is cited, in accordance with accepted academic practice. No use, distribution or reproduction is permitted which does not comply with these terms. 